This document is the accepted manuscript version of the following article: Held, J., Lorimer, T., Albert, C., \& Stoop, R. (2017). Hebbian learning clustering with Rulkov neurons. In G. Mantica, R. Stoop, \& S. Stramaglia (Eds.), Springer proceedings in physics: Vo1. 191. Emergent complexity from nonlinearity, in physics, engineering and the 1ife sciences (pp. 127-141). https://doi.org/10.1007/978-3-319-47810-4_11

\title{
Hebbian Learning Clustering with Rulkov Neurons
}

\author{
Jenny Held ${ }^{* 1}$, Tom Lorimer ${ }^{\dagger 2}$, Carlo Albert ${ }^{\ddagger 1}$, and Ruedi Stoop ${ }^{\S 2}$ \\ ${ }^{1}$ EAWAG, Swiss Federal Institute of Aquatic Science and Technology, 8600 Dübendorf, Switzerland. \\ ${ }^{2}$ Institute of Neuroinformatics and Institute of Computational Science UZH/ETHZ, Irchel Campus 8057 Zurich, \\ Switzerland.
}

June 28, 2019

\begin{abstract}
The recent explosion of high dimensional, high resolution 'big-data' from automated bioinformatics measurement techniques, demands new methods for unsupervised data processing. An essential analysis step is the identification of groups of similar data, or 'clusters', in noisy high-dimensional data spaces, as this permits to perform some analysis steps at the group level. Popular algorithmic clustering algorithms introduce an undesired data shape bias, require prior knowledge of the number of clusters, and are unable to properly deal with noise. Manual data gating, often used to assist these methods, is based on low-dimensional projection techniques, which is prone to obscure the underlying data structure. While Hebbian Learning Clustering successfully overcomes all of these limitations (by using only local similarities to infer global structure), previous implementations were unsuited to deal with big data sets. Here, we present a novel implementation based on realistic neuronal dynamics that removes also this obstacle. By a performance that scales favourably compared to all standard clustering algorithms, unbiased large data analysis becomes feasible on standard desktop hardware.
\end{abstract}

\section{Introduction}

Data analysis begins with detecting structure. High-dimensional big-data has changed the face of this fundamental step. Biological experiments now routinely produce automated high-throughput, highdimensional measurements of unknown elements, which can no longer be feasibly analysed manually. This large data volume requests an unsupervised simplification by a partitioning of the data into groups of similar measurements, which are presumed to manifest on high dimensional structures in the space of measured features. As a result, clustering algorithms have become a standard tool in the analysis of such data sets. The goal of such an algorithm is to infer these structures from similarity between measurements and find classes, or 'clusters', of similar data. However, the global distribution of data in the feature space generally has a highly non-trivial relationship to the point-to-point distances between measurements. An automated clustering procedure therefore faces several difficulties. First, the data may contain clusters of any shape and thus shape biases need to be avoided. In particular, it is well known that stable periodic solutions of non-linear dynamic systems are distributed over convex-concave shrimp-like regions in parameter space $[1,2]$ and that

\footnotetext{
*jenny.held@eawag.ch

†lorimert@ini.uzh.ch

¥carlo.albert@eawag.ch

$\S$ ruedi@ini.uzh.ch
} 
this distribution is preserved in the feature space of the system [3]. Second, the number of clusters is generally unknown and may even change over time. And third, the measurements usually include noise, which asks for a method to classify data as outliers.

Traditional clustering algorithms, such as the partitional k-means [4] and the hierarchical Ward's [5] algorithms, or variations of these, are now widely used in the bioinformatics community $[6,7,8]$. Both methods, however, introduce strong biases that make them infeasible choices for unknown data sets. The k-means clustering procedure, by computing distances between points and representations of sets of points (e.g. cluster centres), disregards the complex relationship between local and global structure and as a result imposes a convex shape bias. Additionally, it fails the remaining requirements as it intrinsically imposes a bias on the number of clusters, which has to be pre-specified, and cannot deal with outliers, since all data items have to be assigned to a cluster. Ward's algorithm, at first sight, seems to avoid biases resulting from to global distance measures, as it constructs a hierarchy of clusters by recursively merging points and groups of points based on their separation. Although at a low hierarchical level this only takes into account local measurements of distance, on higher hierarchical levels it still involves the measurement of global distances between sets of points. This once more prevents the identification of convex-concave shapes, independently of the distance measure used. Similar to the k-means algorithm, outliers cannot be dealt with properly, and the number of clusters remains dependent on the hierarchy level, so that none of the above mentioned criteria for unbiased clustering are met.

In this paper, we provide a solution to these issues. The key to successfully clustering data in an unbiased way is to use local information only, and from this to infer the global data structure. Neural network inspired algorithms, such as Sequential Superparamagnetic Clustering (SSC) [9] and Integrate-and-Fire Hebbian Learning Clustering (I\&F HLC) [10], perform this inference in a natural way, mapping the computational task on a problem addressed in the cortical learning context with the Hebbian learning principle. In the HLC procedure, data items (i.e. points in the space of measured features) are interpreted as neurons with an internal dynamics and the ability to interact with each other via nearest neighbour coupling. Local structure is initially extracted from the data by translating pair-wise distances between data items into similarities. This topological similarity is then converted into dynamical similarity by constructing a network of pair-wise coupled neurons and imposing a coupling strength that is positively correlated with the synchrony between neurons. The Hebbian learning principle, which uses the bio-inspired rule that only the coupling between neurons with highly correlated dynamics is strengthened, can now be used to let the global structure emerge in a self-organised way: The coupling between highly synchronous neurons will be increased at the cost of the coupling strength between less synchronous neurons, so that initial fluctuations in similarity are amplified in such a way that only intra-cluster connections are strengthened. An example of an initial and final state of such a network extracted from an example data set is shown in Figure 1. As a result of this procedure, clusters will emerge as groups of strongly coupled, synchronised neurons. The principle of preferential reinforcement of some connections at the cost of others reflects the fundamental homeostatic tendencies of natural physical systems with sparse resources, and has been linked to the occurrence of power law distributions in the network connectivity and the emergence of community structures in networks at mesoscopic scales [11, 12].

Previous implementations of the HLC, however, were too computationally expensive to be effectively applied to large data sets. Our new implementation of the HLC principle improves upon the I\&F HLC algorithm by making use of the inherently sparse nature of the network as well as the biologically more realistic and lean Rulkov neuron dynamics. Fast nearest neighbour and graph searching algorithms as well as our sparse implementation, using the local nature of the interactions, now allow a linear scaling of computation time with the number of data items, as well as the clustering of data sets with more than $10^{5}$ measurements, which was not possible previously.

We will first describe the structure and behaviour of the algorithm, demonstrate its stability 

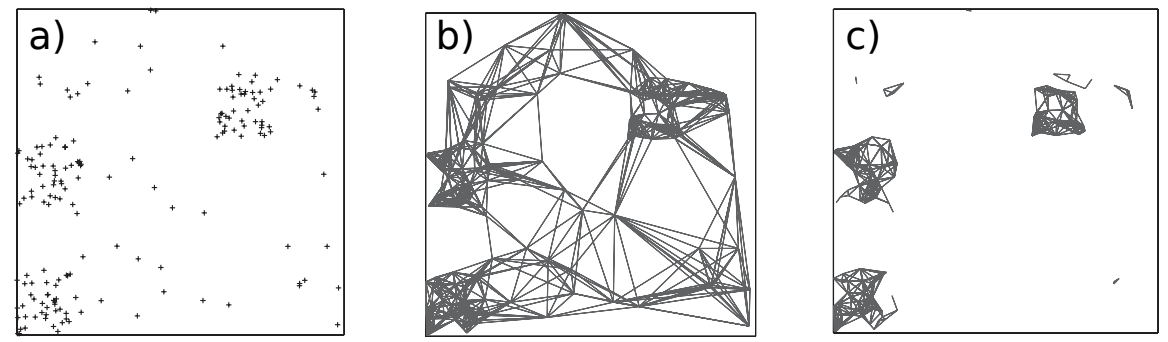

Figure 1: a) A two-dimensional synthetic data distribution. b) The initial HLC network structure. c) Evolved HLC network structure, exposing three main clusters, were points identified as outliers are not shown. [10]

and time performance, and validate its ability to identify clusters without shape bias with several synthetic data sets. We then analyse a biological mass cytometry data set of human bone marrow cells and point out potential drawbacks of manual and standard algorithmic clustering procedures.

\section{The Rulkov Neuron Hebbian Learning Clustering Algo- rithm}

The aim of a clustering algorithm is to extract global structure from local density information in an unbiased way. The HLC succeeds in this by using exclusively local (i.e. point-to-point) topological similarities between data items to impose dynamic similarity between representative neurons, which is then used in the Hebbian learning procedure to identify groups of similar data. In order to achieve this, local structure needs to be extracted from the data first. We can encode this local information in a weighted network that favours connections between data items that are similar, where this similarity is interpreted as pair-wise proximity in the feature space. A natural way to construct such a network from the original set of measurements, with $N$ data items of dimension $d$, is to impose a nearest neighbour coupling. Therefore, as a first step, the $k$ nearest neighbours of each item $i$ are found based on the pair-wise distances $d_{i j}$, where an appropriate distance measure, for instance Euclidean distance, is chosen. Thus, a sparse non-symmetric adjacency matrix $\mathbf{A}$ is constructed, where $A_{i j}=1$ if $j$ is a nearest neighbour of $i$ and $A_{i j}=0$ otherwise. An example 2D data set and the graph representing the initial adjacency of points are shown in Figure 1a) and b) respectively, where the direction of the connections is omitted. A weighting that encodes pair-wise similarity can then be realised in the choice of an initial coupling strength $g_{i j}$ between the two neurons, at time $t=0$ :

$$
g_{i j, t=0}=A_{i j} \exp \left[-\epsilon\left(\frac{d_{i j}}{d_{0}}\right)^{2}\right],
$$

where $d_{0}$ is the average distance between all neighbours in the network and $\epsilon \in \mathbb{R}_{>0}$ is a scaling constant. A different measure that results in an increase in similarity for decreasing distance may also be feasible.

The similarity encoded statically in these weights can now be translated into a dynamic similarity. To this end, the data items are interpreted as neurons with internal dynamics, on which the coupling imposes dynamic correlation, or 'synchrony'. The input to a neuron, which is coupled only to its neighbours, also contains information about the dynamics of more distant parts of the network, mediated by the dynamics of structures of neurons connecting these parts. Thus, intermediate- and 
large-scale synchronisation patterns may emerge even though not all neurons within these groups are coupled directly. In order for the final weight structure to represent these large-scale correlations, Hebbian learning is used to adjust the weights to represent the 'true' similarity between data points. In this process, the neuron group interactions allow for initial local variations in similarity to be either amplified or smoothed out. For instance, groups of neurons may collectively behave synchronised despite small variations in the weights, so that all intra-group connections may be strengthened. On the other hand, the lack of similar driving inputs will cause neurons from different groups or outliers to behave less similarly, so that their initial dissimilarity can be amplified in the learning process. The final weight structure, as shown for instance in Figure 1c), therefore essentially represents global dynamic similarity, as opposed to topological proximity.

Here, the dynamics imposed on the nodes is given by the Rulkov neuron model [13], which in contrast to the I\&F model used in earlier implementations is realistic in the sense that it is capable of producing any real-life neuron behaviour, including spiking, bursting, and silent phases. Each node has two time-discrete state variables, a fast evolving (membrane) potential $x_{i, t}$ and a slowly evolving (phase) potential $y_{i, t}$, given by

$$
\begin{aligned}
& x_{i, t+1}=f\left(x_{i, t}, y_{i, t}+\beta_{i, t}\right), \\
& y_{i, t+1}=y_{i, t}-\mu\left(x_{i, t}+1\right)+\mu\left(\widetilde{\sigma}_{i}+\sigma_{i, t}\right),
\end{aligned}
$$

where $\widetilde{\sigma}_{i}$ represents a constant input current driving the uncoupled neuron, and the evolution of the fast variable is given by

$$
f(x, y)=\left\{\begin{array}{lll}
\frac{\alpha}{1-x}+y & \text { if } & x \leq 0 \\
\alpha+y & \text { if } & 0<x<\alpha+y \\
-1 & \text { if } & x \geq \alpha+y
\end{array}\right.
$$

Nodes can interact via the Rulkov coupling equations, with a strength proportional to the coupling $g_{i j, t}$ :

$$
\begin{aligned}
\beta_{i, t} & =\beta_{c} \frac{1}{n} \sum_{j=1}^{n} g_{i j, t}\left(x_{j, t}-x_{i, t}\right), \\
\sigma_{i, t} & =\sigma_{c} \frac{1}{n} \sum_{j=1}^{n} g_{i j, t}\left(x_{j, t}-x_{i, t}\right),
\end{aligned}
$$

where the parameters $\beta_{c}, \sigma_{c} \in[0,1]$ are constants that may be used to weight the influence of the coupling on the fast and slow potentials.

The currents $\beta_{i, t}$ and $\sigma_{i, t}$ allow the neurons to communicate and synchronise for large coupling strengths. In the Hebbian learning procedure, this dynamic similarity needs to be quantified. It is essential that such a measure of synchrony and the coupling strength between neurons interact appropriately. This includes the direction of change, i.e. an increase in synchrony as a result of an increase in coupling strength (and vice versa), and a preferably small time scale on which the synchrony reacts to a change in coupling. These requirements are realised in a measure of phase synchrony, where the synchrony $S_{i j, t}$ at time $t$, measured over a preceding time period $\tau$, is given by means of the population Pearson correlation between the slow potentials of the neurons: 


$$
S_{i j, t}=\frac{\sum_{\ell=t-\tau}^{t}\left(y_{i, \ell}-\bar{y}_{i}\right)\left(y_{j, \ell}-\bar{y}_{j}\right)}{\sqrt{\sum_{\ell=t-\tau}^{t}\left(y_{i, \ell}-\bar{y}_{i}\right)^{2} \sum_{\ell=t-\tau}^{t}\left(y_{j, \ell}-\bar{y}_{j}\right)^{2}}},
$$

where $\bar{y}_{i}$ is the average potential of the $i$ th neuron over the period $\tau$.

A single Hebbian update rule, that comprises both a tendency for global synchronisation and a restriction of the same due to resource sparsity, can now be realised. These two counteracting principles, mediated locally, eventually result in the formation of global and mesoscopic structures, from which clusters can be extracted. With this aim, the learning rule is formulated such that the coupling between two neurons will be increased or decreased depending on the nodes' synchrony relative to the average synchrony in the network, such that high synchrony results in an increase in coupling and low synchrony in a decrease:

$$
g_{i j, t+1}=g_{i j, t}+\frac{\tau}{t}\left(c_{1} S_{i j, t}-c_{2} \bar{S}\right),
$$

where $c_{1}, c_{2} \in[0,1]$ and $\bar{S}$ is the average synchrony in the network, given by

$$
\bar{S}=\sum_{i, j \in \mathbb{N}, A_{i j}=1} S_{i j, t} .
$$

An example of the development of some weights between different neurons is shown in Figure 2. It can be seen that the weights settle to an equilibrium where only neurons within a cluster will maintain their strong connections.
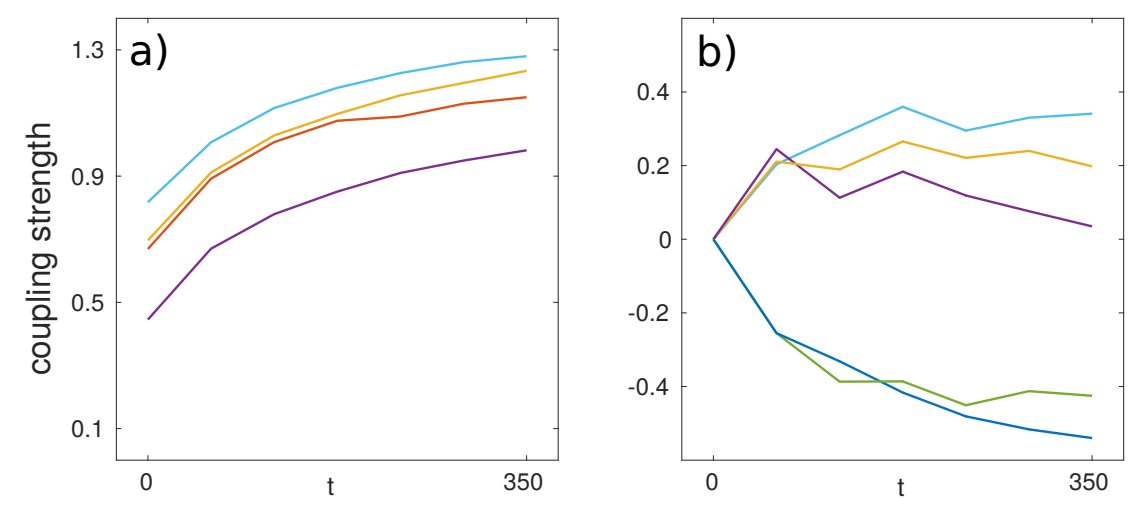

Figure 2: a) Development of several weights within a cluster, where all weights are increased in the Hebbian learning procedure. b) Development of weights among low-density noise data (top three weights) and the weights with neurons from an adjacent cluster. The weights among noise data are increased initially, due to the fact that the nodes are in an environment of similar density, but overall remain small in compensation for the increase in coupling strengths within clusters.

Finally, the clusters are to be extracted from the resulting global weight structure. Towards the end of the learning process, the system will be driven into state where the number of strong weights does not change significantly any more (i.e. all strong connections are found) while the 
number of intermediate-strength connections becomes small (due to a strong increase or decrease in the learning procedure). That is, it has become clear whether two neighbours do have a strong correlation or not. At this stage, the final structure is found and the learning procedure can be stopped. To determine when this stage is reached, a stopping coefficient $\kappa$, which is given by the ratio of still-learning connections $n_{\ell}$ to the total number of strong connections $n_{s}$ in the network, is defined:

$$
\kappa=\frac{n_{\ell}}{n_{s}} .
$$

The number of connections that are still learning is determined using two thresholds $\theta_{1}$ and $\theta_{2}$. Stilllearning connections are said to have weights in the intermediate range with $\theta_{1}<g_{i j}<\theta_{2}$, while strong connections have a high coupling strength $g_{i j}>\theta_{2}$. The learning process is stopped when $\kappa$ falls below a small threshold, $\kappa \leq \omega$, indicating a low number of still-learning weights compared to already settled weights.

In the final step, clusters are defined as structures of strongly coupled nodes. These can be extracted from the network as subgraphs of nodes with weights above a certain threshold. In our implementation, this is done by applying the fast Tarjan's algorithm [14] in the graph of weights above threshold $\theta_{2}$.

\section{Stability and Computational Complexity of the RHLC}

\subsection{Stability of the RHLC Results}

While the numerical values of some parameters used in the algorithm have biological or computational motivation and the algorithm is stable towards changes in others, mainly two parameters directly influence the clustering result and may be used for tuning. These are the coupling initialisation parameter $\epsilon$ and the number of nearest neighbours $k$. For a synthetic data set, where the ideal clustering result is known, we can measure the quality of any RHLC clustering for ranges of these parameters. In Figure 3, we present an example outcome of such an experiment, where we measure the quality of a clustering result using the Jaccard index $([15,16]$, see appendix), a measure of similarity between sets, where an index close to 1 indicates a good clustering result.

It is clear that there is a region of stability, where changes in the parameters do not alter the clustering result significantly However, with more extreme values, a preference for larger or smaller clusters can be induced, as shown in Figures 3c) and f). A large number of nearest neighbours or a low value of $\epsilon$, which increases the average similarity between neurons, favours larger clusters, whereas low similarity and few neighbours effectively splits clusters up into smaller clusters. A hierarchy becomes apparent, and the naturalness of a cluster can be inferred from its stability, where the most natural clusters will be the most robust with respect to changes in these parameters.

The numerical values of the other parameters and initial conditions used in this implementation are motivated by simulations with toy data sets or theoretical arguments, and are presented for completeness in the appendix.

\subsection{Computational Complexity of the RHLC}

Our HLC implementation makes use of the computationally lean (discrete) Rulkov neuron dynamics and the local nature of the interactions (and therefore sparse form of the interaction matrices), and is thus able to achieve a favourable scaling of computation time with respect to the number of data items. From Figure 4, a practical estimation of the complexity of the RHLC (Matlab implementation) reveals a scaling of $\approx O(N)$, similar to the k-means algorithm (Matlab implementation) 

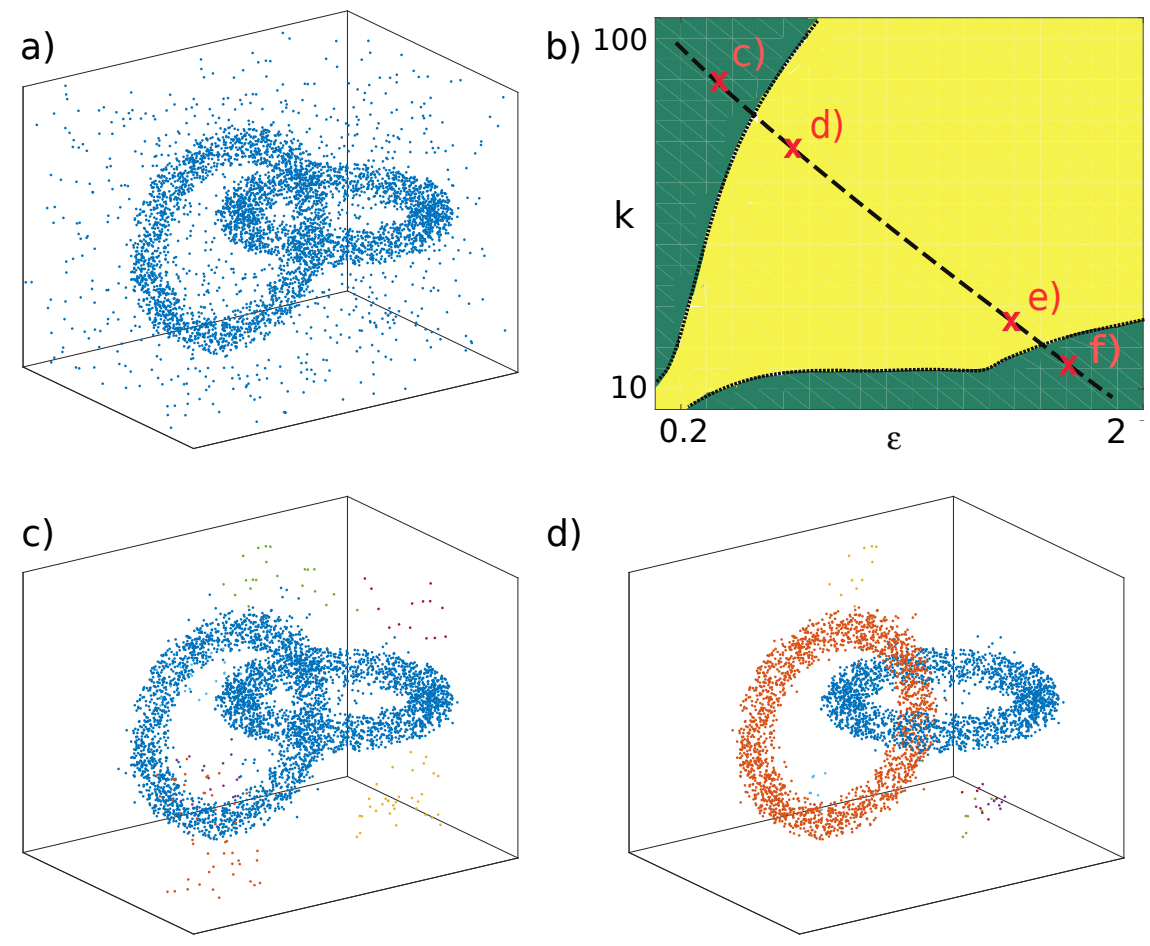

d)
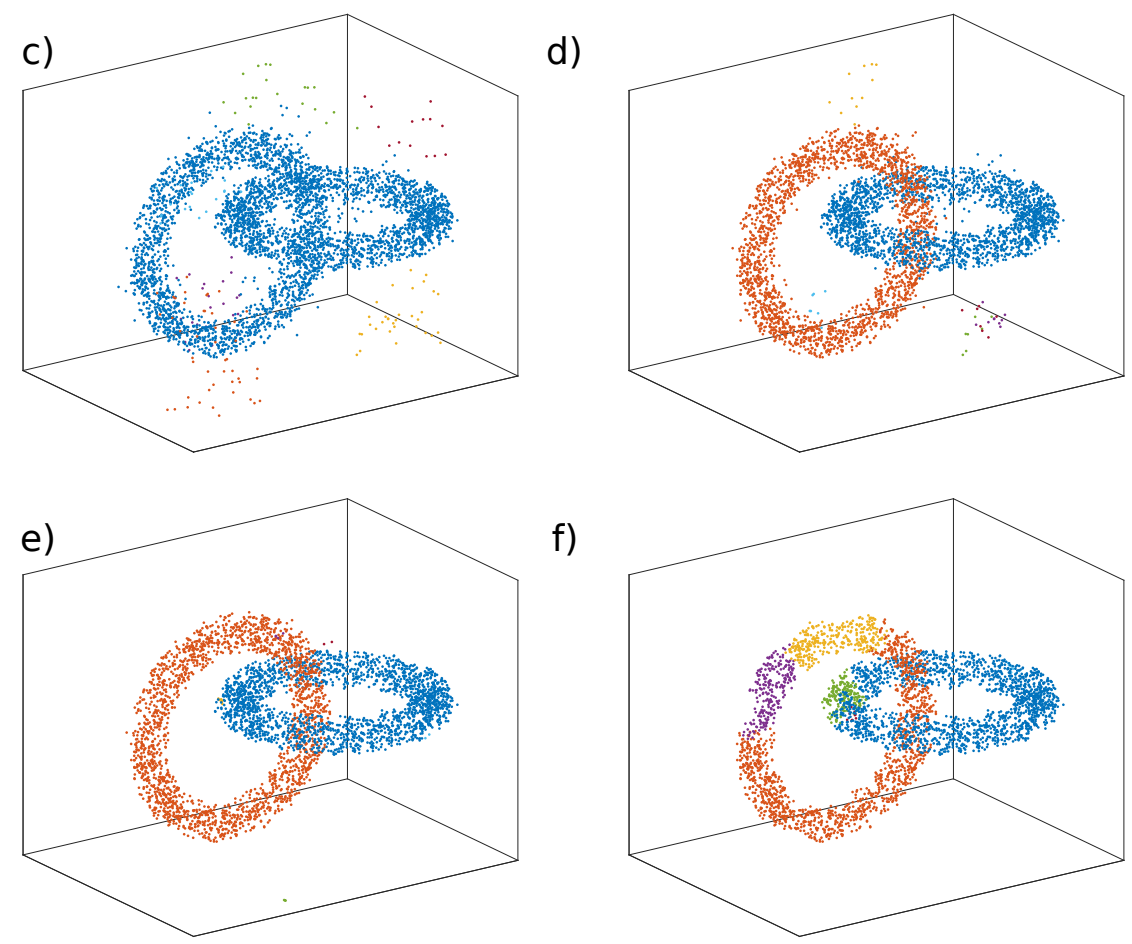

f)

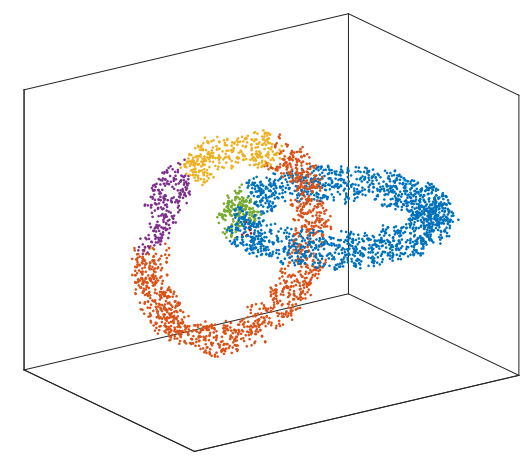

Figure 3: a) A synthetic 3D data set with two intertwined rings that represent convex-concaveshaped clusters, and background noise data, simulating outliers. b) The clustering result quality as function of the parameters $k$ and $\epsilon$, where a Jaccard index close to 1 (yellow region, points d) and e)) marks a good clustering result, while low Jaccard indices (green region, points c) and f)) indicate poor results. In the top left parameter region a tendency towards large clusters is observed, in the bottom right region a tendency towards small clusters, where the specific results are displayed in Figures c)-f) respectively.

and the k-means related algorithm 'flowPeaks' ( $\mathrm{R}$ implementation) [8], which is used in the flow cytometry community. The I\&F HLC (C implementation, of which no sparse version exists at this time) and Ward's algorithm (Matlab implementation) show a scaling of $\approx O\left(N^{2}\right)$.

It is clear that the HLC algorithms have an offset in computation times compared to classical al- 
gorithms, due to the network initialisation and repeated computation of the neuron potentials and synchrony, but that due to the local nature of interactions, a linear scaling can be achieved. In the RHLC algorithm, the $k$-nearest neighbour search requires $O(k N \log (N))$ and the connected components search $O(N+k N)$. The computation of synchrony and the weight updates occur with $O(k N)$, multiplied by the number of weight updates required until the learning procedure is stopped. With our learning rule and the Rulkov neuron dynamics, we were able to reduce the number of updates required by a factor of $\approx 0.5$ compared to the I\&F HLC. The limiting factor for very large data sets will thus be the network initialisation. The local interaction rules, however, also open up the possibility of parallelisation, which would further reduce the total computation times and therefore the computation time offset.

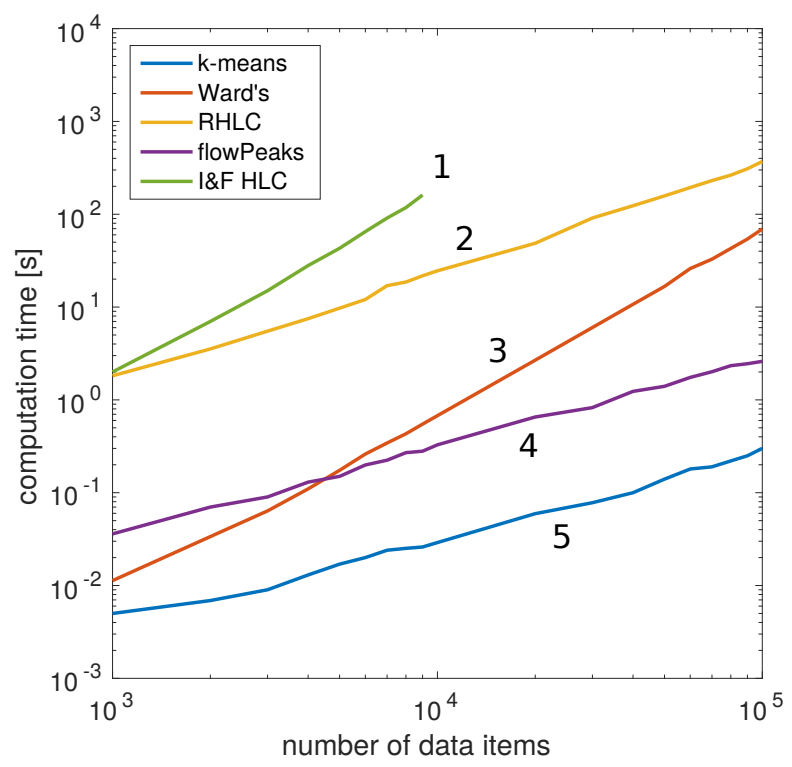

Figure 4: Computation times of several clustering algorithms. The estimated slope values indicate a favourable behaviour of the RHLC (2) which scales with $O(N)$, similar to the flowPeaks (4) and k-means (5) algorithms. The I\&F HLC (1) and Ward's (3) algorithms scale with $O\left(N^{2}\right)$. The offset of the HLC algorithms due to the network initialisation and repeated neuron potential computation may be reduced further by parallelisation. 


\section{Application to Data Sets}

We first analyse a synthetic data set that is ideal for testing the requirements for an unbiased clustering algorithm posed in the beginning. The data set, shown in Figure 5a), contains two convex-concave shapes of different sizes as well as noise data. The dataset illustrates the complex relationship between local and global similarity, where the membership of a point to a cluster is mediated by local connectivity, not necessarily global proximity. As a result it can be seen in Figure 5b) that Ward's algorithm, which implicitly uses the assumption of global proximity, is unable to identify the clusters, while the RHLC finds them in a natural way (Figure 5c)). While parts of the long extended 'legs' of the clusters are identified as separate clusters, due to larger gaps between segments, its ability to extract clusters of any shape from local density measures is evident.
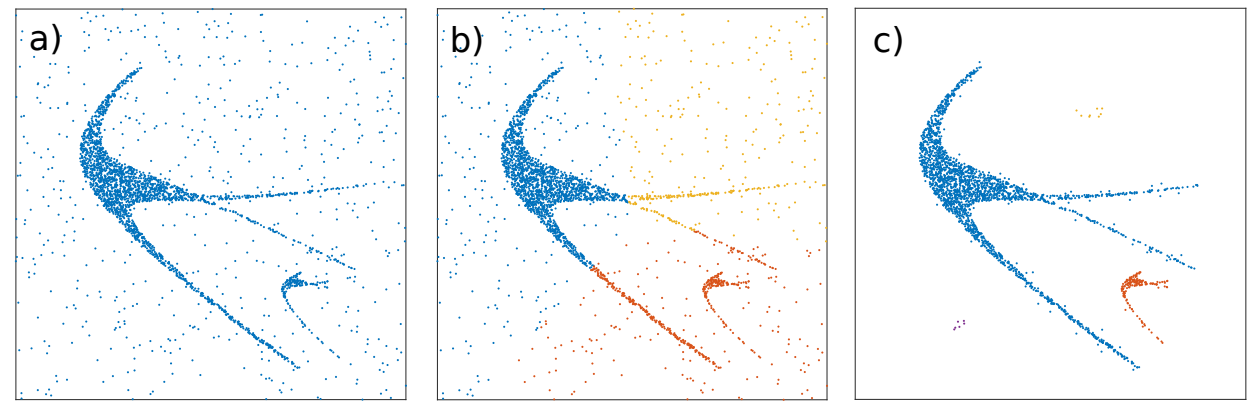

Figure 5: a) The initial 2D convex-concave synthetic data set, with background noise data items. b) Ward's clustering result, where the clusters are not identified correctly. c) With the RHLC, a close-to-ideal clustering result is found.

A promising real-life application of our automated clustering approach is the identification of cell types. Many diseases, such as acute myeloid leukemia, manifest themselves in functionally and phenotypically diverse cells, the classification of which is essential for disease identification and relapse prevention. Human bone marrow, containing a variety of distinct and well-characterized immunological cell types, presents an ideal benchmark case for phenotypic clustering [17]. We therefore analyse here a mass cytometry dataset of healthy human bone marrow cells (data and description of experimental method: [18]). A standard method for cell type identification in mass cytometry data is an expert manual gating procedure, where constant thresholds are introduced in biaxial plots to separate classes of cells. Here, we compare the results of this manual low-dimensional gating procedure with the results of the analysis in higher dimensions with Ward's and the RHLC algorithms. In the analysis, 13 different surface marker expressions were used as cell features. These are the integrated elemental reporter signals recorded in a time-of-flight mass cytometer, induced by transition element antibody tags labelled with elemental isotopes. For the visualisation of the results, t-SNE projections [19] have become a standard tool, allowing the mapping of high dimensional data to a low-dimensional space while aiming to preserve relative inter-item distances.

The classification results are presented in Figure 6, where for Ward's clustering the manually found number of clusters was supplied. Several differences between the results become apparent. First, Ward's algorithm fails to correctly identify several clusters and tends to erroneously split up clusters that are identified by the RHLC and manual gating as one cluster.

Furthermore, the results also raise questions about the expert labelling procedure. The RHLC clustering generally agrees well with the manual clustering, but merges some of the clusters that are distinguished in the manual gating (such as the three bordered clusters in Figure 6a), which become a single cluster in the RHLC clustering, as shown in Figure 6c)). 

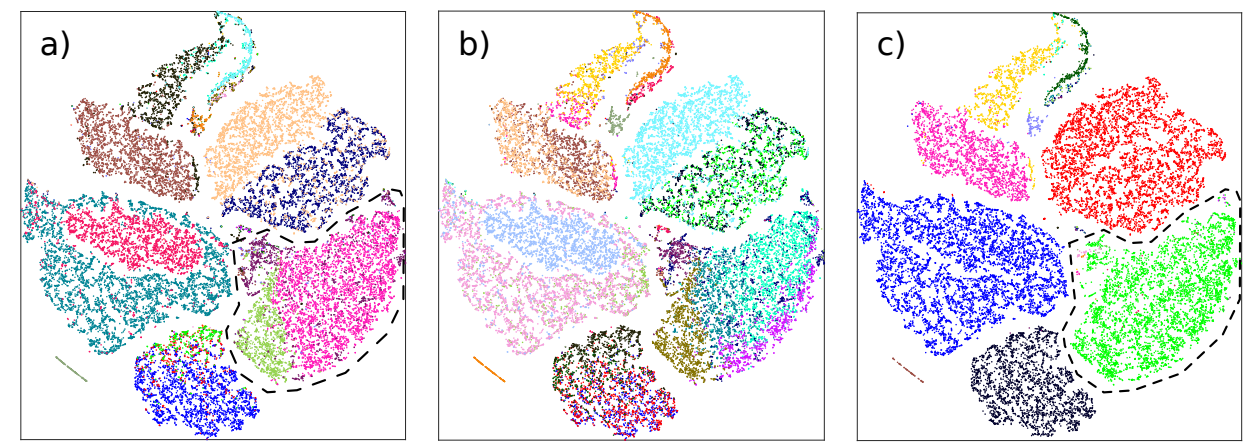

Figure 6: The t-SNE projections [19] of 30000 cells from healthy human bone marrow benchmark data [18], where colours encode cluster labels.

a) Expert cluster labels, which were found by biaxial manual gating [18]. b) Ward's cluster labels, where the ideal number of clusters (24) was supplied. c) Most stable RHLC clustering result. While Ward's clustering is very sensible to small separations and density gradients, the very stable RHLC result suggests generally larger clusters.
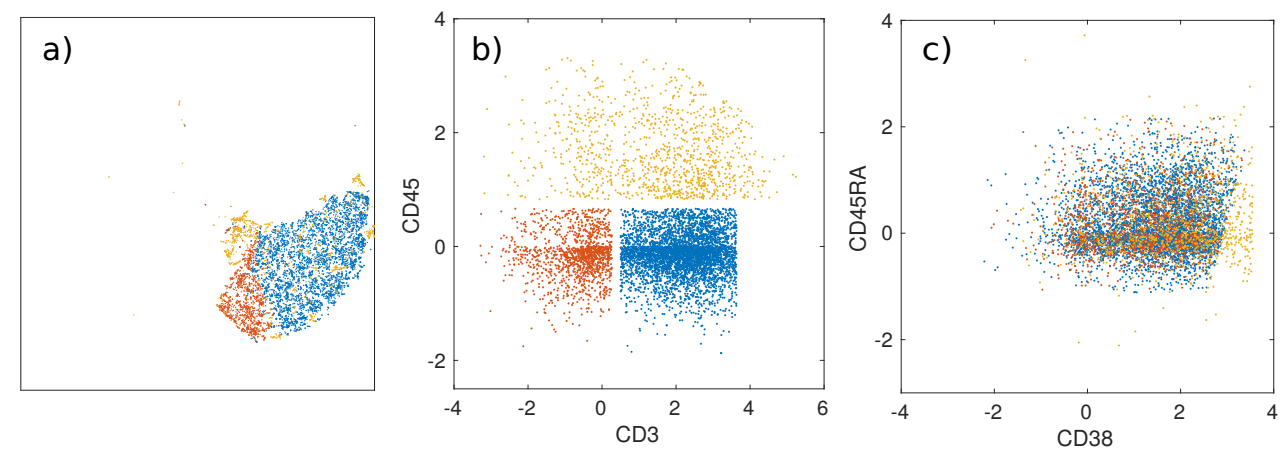

Figure 7: a) The t-SNE map of three manually labelled clusters. b) The two-dimensional projection of the same clusters in the only two separating dimensions. c) In all other dimensions, the clusters occupy roughly the same ranges, such as demonstrated with these example features. Here, the standard mass cytometry data transformation, asinh of the data divided by 5 , was applied.

Searching for the features that give rise to the distinction between these clusters in the manual gating procedure, we look at the two-dimensional orthogonal projections of the data in Figure 7 . It becomes clear from these projections that the distinction between the clusters originates from very thin, linear, separating gaps between the clusters in a single dimension, such as one would expect to be introduced in a thresholding procedure, where piecewise linear boundaries are used to define cell clusters. All major differences between the manual and the RHLC classifications can be accounted for by such gaps in different dimensions. These gaps may be introduced in the manual gating procedure or some data pre-processing step, and naturally make appealing targets for manual gating as well as hierarchical algorithms such as Ward's algorithm. However, these two-dimensional projections may be misleading. Comparing the size of the gaps with the average nearest neighbour distance in the high-dimensional space, we find that the size of the gap $(d \approx 0.1$ in the transformed coordinates) is small compared to the average nearest neighbour distances in the respective clusters $(d \approx 1)$. Using the RHLC, we find that the most stable clustering configuration is one where these clusters are merged. Although the RHLC can be tuned to separate these clusters as well, the stability of the 
result shown in Figure 6c) suggests that the separation in the single dimensions is not significant compared to the overlap in other dimensions. Questions about the biological interpretation and motivation behind the thresholding procedure, as well as the interpretation of the RHLC result, arise and will be investigated in the future.

\section{Conclusions}

We presented the basic principles, implementation, and results of a neuronal network based clustering algorithm that combines the robust, self-organised design of the Hebbian learning clustering with the biologically realistic Rulkov neuron dynamics and an efficient sparse implementation. By using unsupervised learning in an artificial neural network based on local interactions, global information about the cluster membership can be extracted avoiding the shape biases of standard clustering algorithms. Additionally, its hierarchical response to changes in certain parameters allows the evaluation of the stability and thus naturalness of a cluster.

We demonstrated the algorithm's ability to identify convex-concave clusters in the presence of noise data with several synthetic data sets. For a high-dimensional biological bone marrow data set, we showed that the RHLC, by using information from all dimensions simultaneously, may provide insights that are not accessible by manual clustering procedures.

Our sparse implementation now allows the fast analysis of large data sets, where the possibility for further optimisation by parallelisation is given. This opens up new possibilities to analyse large, high-dimensional, unknown data sets, without prior knowledge about the number, shape, or density of clusters.

We note that further deparametrisation may be possible by altering the Hebbian learning rule to include a local measure of the average synchrony, so that it is of the form $g_{i j, t+1}=g_{i j, t}+\frac{\tau}{t}\left(S_{i j, t}-\bar{S}_{i}\right)$, where $\bar{S}_{i}$ is the average synchrony in the neighbourhood of neuron $i$ only. This would complement the algorithms local nature and provide the possibility of a further increase in stability.

\section{Appendix}

\section{Parameters and Initial Conditions}

The initial potentials of the neurons are randomised around $\left\{x_{i}, y_{i}\right\}=\{-1,-3\}$, which are values that a standard uncoupled neuron will visit naturally in its cycle. The algorithm is stable with respect to this random initialisation. The parameter ranges that may in principle be used are presented in Table 1.

\begin{tabular}{cll}
\hline Parameter & Range & Description \\
\hline$k$ & $\{1,2, \ldots, N-1\}$ & number of nearest neighbours \\
$\epsilon$ & $\mathbb{R}_{>0}$ & coupling initialisation parameter \\
lightgrayheight $\beta_{c}, \sigma_{c}$ & {$[0,1]$} & Rulkov neuron dynamical parameters \\
$\mu$ & $(0,1)$ & Rulkov neuron dynamical parameter \\
$\alpha$ & $\mathbb{R}_{>0}$ & Rulkov neuron dynamical parameter \\
lightgrayheight $\tau$ & $\mathbb{N}$ & time over which synchrony is measured \\
$\theta_{1}, \theta_{2}$ & $\mathbb{R} \mid \theta_{1}<\theta_{2}$ & thresholds for weak and strong weights \\
$c_{1}, c_{2}$ & {$[0,1]$} & weight update parameters \\
$\omega$ & $\mathbb{R}_{>0}$ & stopping criterion \\
blackheight & &
\end{tabular}

Table 1: Parameters and their ranges in the Rulkov HLC algorithm. 
We find that generally, the best results are obtained with the initialisation parameters $k \in$ $\{10 \ldots 100\}$ and $\epsilon \in[0.5,2]$, where the clustering result is in general stable for ranges of these parameters, as shown in Figure 3. The Rulkov neuron parameters are set to allow all types of neuron dynamical behaviours, a fast evolution of the potentials, and non-weighted coupling $(\alpha=8$, $\mu=0.1$, and $\beta_{c}, \sigma_{c}=1$ ). The parameter $\tau$ is chosen $\tau=50$, which was found to be the minimum time required for the synchrony to be measured reliably. Higher values are equally suitable but increase the computation time. The parameters $c_{1}$ and $c_{2}$ were chosen $c_{1}=\frac{1}{2}$ and $c_{2}=\frac{1}{3}$ to allow for a global increase in coupling strength in the initially generally weakly coupled network. The thresholds $\theta_{1}$ and $\theta_{2}$ define what is a weak, respectively strong, connection. The parameters $\theta_{1}$ and $\theta_{2}$ encode the interpretation of the strength of a coupling. Two Rulkov neurons generally behave synchronously for large values of $g_{i j}$ and less synchronous for low values, so here we choose $\theta_{1}=0.2$ and $\theta_{2}=0.9$. The value of $\omega$ has to be chosen small to ensure that the number of still-learning connections has become small enough compared to the number of already-settled connections. In our implementation, motivated by experiments with toy data sets, we choose $\omega=0.4$.

The constant parameters $\widetilde{\sigma}_{i}$ are computed by

$$
\widetilde{\sigma}_{i}=\frac{G_{i}}{\max _{i} G_{i}}
$$

where the total coupling strength $G_{i}$ of a neuron is given by the sum over all initial coupling strengths to all its neighbours, normalised by the number of neighbours,

$$
G_{i}=\frac{1}{k} \sum_{j=1}^{k} g_{i j, 0}
$$

This initialisation of $\widetilde{\sigma}_{i}$ ensures that neurons in similarly dense regions have a similar constant input current and tend to behave synchronously from the beginning.

\section{Jaccard similarity}

The Jaccard index is for two clusterings $c_{r}$ and $c_{o}$ is given by

$$
J\left(c_{r}, c_{o}\right)=\frac{a\left(c_{r}, c_{o}\right)}{a\left(c_{r}, c_{o}\right)+e\left(c_{r}, c_{o}\right)+e\left(c_{o}, c_{r}\right)},
$$

where $a\left(c_{r}, c_{o}\right)$ is the number of observation pairs that are in the same cluster in $c_{r}$ as well as $c_{o}$, and $e\left(c_{r}, c_{o}\right)$ is the number of observation pairs that are in the same cluster in $c_{r}$ but not in $c_{o}$. The Jaccard index is thus large $\left(J\left(c_{r}, c_{o}\right) \approx 1\right)$ for high similarity between the clusterings, and low $\left(J\left(c_{r}, c_{o}\right) \approx 0\right)$ for low similarity. Therefore, if a given clustering result $c_{r}$ is compared to some optimal clustering result $c_{o}$, a Jaccard index close to 1 indicates a good clustering result. 


\section{References}

[1] Stoop R., Martignoli S., Benner P., Stoop R.L., Uwate Y. (2012) Shrimps: Occurrence, scaling and relevance. Intl. J. Bif. \& Chaos, 22(10), 1230032.

[2] Stoop R., Benner P., Uwate Y. (2010) Real-world existence and origins of the spiral organization of shrimp-shaped domains. Phys. Rev. Lett., 105, 074102.

[3] Gomez F., Stoop R.L., Stoop R. (2014) Universal dynamical properties preclude standard clustering in a large class of biochemical data. Bioinformatics, 30(17), p.1-8.

[4] McQueen J.B. (1967) Some methods for classification and analysis of multivariate observations. Proc. Fifth Berkeley Symp. on Math. Statist. and Prob., 1, p.281-297.

[5] Ward J.H. Jr. (1963) Hierarchical grouping to optimize an objective function. J. Am. Stat. Assoc., 58(301), p.236-244.

[6] Wong D.S.V., Wong F.K., Wood G.R. (2007) A multi-stage approach to clustering and imputation of gene expression profiles. Bioinformatics, 23(8), p.998-1005.

[7] Bréhélin L., Gascuel O., Martin O. (2008) Using repeated measurements to validate hierarchical gene clusters. Bioinformatics, 24(5), p.682-688.

[8] Ge Y., Sealfon S.C. (2012) flowPeaks: a fast unsupervised clustering for flow cytometry data via k-means and density peak finding. Bioinformatics, 28(15), p.2052-2058.

[9] Ott T. et al. (2004) Sequential Superparamagnetic Clustering for Unbiased Classification of High-Dimensional Chemical Data. J. Chem. Inf. Comput. Sci., 44(4), p.1358-1364.

[10] Landis F., Ott T., Stoop R. (2010) Hebbian self-organizing integrate-and-fire networks for data clustering. Neural Computation, 22(1), p.273-288.

[11] Gutiérrez R. et al. (2011) Emerging meso- and macroscales from synchronization of adaptive networks. Phys. Rev. Lett., 107, 234103.

[12] Lorimer T., Gomez F., Stoop R. (2015) Two universal physical principles shape the power-law statistics of real-world networks. Scientific Reports, 5, 12353.

[13] Rulkov N.F. (2002) Modeling of spiking-bursting neural behavior using two-dimensional map. Phys. Rev. E, 65(4), 041922.

[14] Tarjan R.E. (1972) Depth first search and linear graph algorithms. SIAM Journal on Computing, 1(2), p.146-160.

[15] Jaccard P. (1902) Lois de distribution florale dans la zone alpine. Bull. Soc. Vaud. Sci. Nat., 38(144), p.67-130.

[16] Jaccard P. (1912) The distribution of the flora in the alpine zone. New Phytologist, 11(2), p.37-50.

[17] Levine et al. (2015) Data-Driven Phenotypic Dissection of AML Reveals Progenitor-like Cells that Correlate with Prognosis. Cell, 162(1), p.184-197.

[18] Bendall et al. (2011) Single-cell mass cytometry of differential immune and drug responses across a human hematopoietic continuum. Science, 332(6030), p.687-696.

[19] Van der Maaten L., Hinton G. (2008) Visualizing Data using t-SNE. J. Mach. Learn. Res., 9, p.2579-2605. 